\title{
Scepticisme et dialectique des Lumières chez le jeune Hegel $^{1}$
}

\author{
Italo Testa
}

(pre-print: published in S. Charles, P. Junqueira-Smith (a cura di), Scepticism in the Eighteenth Century: Enlightenment, Lumières, Aufklärung, International Archives of the History of Ideas / Archives internationales d'histoire des idées, Volume 210, Springer, Heidelbergh/New York/Berlin, 2013, pp 281-297)

\section{Introduction}

En 1802, pendant la deuxième année de son séjour a Iéna (1801-1807), Hegel publie un article sur Le rapport entre le scepticisme et la philosophie qui révèle une très marquante connaissance historique du scepticisme ancien et modern, une réflexion très approfondie sur les problèmes épistémologiques posés par le scepticisme, et une théorie originale - et qui ne changera pas dans ses ouvres suivantes - de la supériorité du scepticisme ancien - en tant que théorie et pratique critique des tropes - sur le scepticisme modern, ce dernier étant entendu comme une forme de dogmatisme de la subjectivité. Selon Hegel le scepticisme ancien, qui trouve sa génuine expression dans le principe de l'antinomie, représente en tant que critique des déterminations positives la face négative de la raison et de toute vraie philosophie ${ }^{2}$. Comment-est il arrivé que Hegel ait développé cette conception rationaliste du scepticisme et de son rôle critique en tant que moment négative de la philosophie? Si la littérature critique sur Hegel et le scepticisme à bien étudié le lien entre cet écrit et les ouvres systématiques, en analysant nombreuses sources philosophiques de l'intérêt de Hegel pour le scepticisme ${ }^{3}$, il me semble que la genèse de la question du scepticisme dans les travaux de jeunesse doit être encore dévoilée. Quel est le lien entre les écrits de jeunesse (1785-1800), qui étaient dédiés à des sujets pratiques, politiques et religieuses, et ce que Hegel écrit en 1802 sur le scepticisme en tant que coté négative de la raison philosophique? Est-ce que la conception épistémologique et métaphilosophique du scepticisme développée a Iéna est une réponse à des problématiques sociales, politiques et religieuses qu'Hegel s'était déjà posé dans les écrits des années précédents ? Et peut être ça pourrait nous révéler d'autres cotés de la problématique sceptique chez Hegel, c'est-à-dire ses dimensions politiques et religieuses? En essayant de répondre à ces questions ouvertes, on rencontre, selon la thèse que je vais exposer, le problème des Lumières chez Hegel. La question de la signifiance des Lumières pour le jeune

\footnotetext{
${ }^{1}$ Je remercie Sébastien Charles et Claudie Hamel pour la révision du texte français.

${ }^{2}$ Cfr. G.W.F. Hegel, Verhältniss des Skeptizismus zur Philosophie, in Gesammelte Werke, Bd. 4: Jenaer Kritische Schriften, hrsg. v. H. Buchner und O. Pöggeler, Hamburg, Meiner, 1968 (désormais cité GW4), p. 208.

${ }^{3}$ Dans le travail le plus important sur le sujet du scepticisme chez Hegel il n’ya que des rares allusions aux écrits de jeunesse (voir M.N. Forster, Hegel and Skepticism, Harvard University Press, Cambridge, Mass., 1989, p. 118-119). Sur le rapport avec le platonisme et le néoplatonisme, voir H. Bucher, Zur Bedeutung des Skeptizismus beim jungen Hegel, «Hegel-Studien», Beiheft 4, Bouvier, Bonn, 1965, p. 49-56; M. Baum, Die Entstehung der hegelschen Dialektik, Bouvier, Bonn, 1989, p. 185-191; F. Chiereghin, Platonische Skepsis und spekulatives Denken bei Hegel, in Skeptizismus und spekulatives Denken in der Philosophie Hegels, hrsg. V. H.F. Fulda und R.-P. Horstmann, Klett-Cotta, Stuttgart, 1997, p. 29-49. Sur d'autres sources sceptiques voir en particulier K. Vieweg, Philosophie des Remis. Der junge Hegel und 'das Gespenst des Skeptizismus', München, Fink, 1999.
} 
Hegel (1785-1800) est en fait directement liée au moment historique et théorique pendant lequel le scepticisme devint constitutif de sa conception de la philosophie.

Cet article abordera deux questions principales: la question de la signification accordée aux Lumières par le jeune Hegel (1785-1800) et la question du moment historique et théorique à partir duquel le scepticisme religieux et épistémologique devient constitutif de la conception hégélienne de la philosophie. Je pense que si nous posons correctement ces questions, il sera possible de reconstruire le parcours du jeune Hegel de façon à éclairer la genèse de sa pensée en identifiant les expériences historiques et théorétiques qui fondent la conception du rapport entre philosophie et scepticisme développée dans l'écrit de 1802. Sur ce point, j'avancerai les thèses suivantes : (1) au cours de sa période de jeunesse, à Berne en particulier (1793-1797), Hegel fait l'expérience du retournement de la raison éclairée ; (2) ce retournement signifie pour Hegel une expérience réelle et intellectuelle de la dialectique des Lumières; (3) l'expérience de la dialectique des Lumières est l'origine historique et théorétique qui a mené Hegel à développer explicitement, dans les années suivantes, une conception dialectique de la pensée et de la réalité ; (4) enfin, l'expérience du retournement dialectique de la raison éclairée a conduit Hegel à soumettre la raison des Lumières, et en particulier sa version kantienne, à l'autoréflexion, cette dernière menant à une autocritique sceptique de la raison qui révèle la structure gnoséologique dualiste du criticisme : la critique des Lumières se transforme ainsi en critique de type sceptique épistémologique.

Ma reconstruction du chemin du scepticisme chez le jeune Hegel se développe donc en quatre étapes. Dans une première étape, j'aborderai la question du scepticisme religieux du jeune Hegel envers l'autorité dogmatique judéo-chrétienne, scepticisme qui a été inspiré par les Lumières, la Révolution française et le républicanisme et se développé politiquement comme critique de la domination. Dans la deuxième étape, je parlerai de l'expérience hégélienne de la dialectique des Lumières qui se tournent en dogmatisme et donc de la critique sceptique rationnelle de la positivité historique et du scepticisme fidéiste qui se lie à elle. Dans la troisième étape, j'aborderai le sujet de l'esprit d'unité en tant que modèle de la logique de la domination qui a été retracée par Hegel dans l'anthropologie judéo-chrétienne et qui se relie à sa conception du destin, dans laquelle scepticisme religieux et scepticisme épistémologique se croisent pour la première fois. Dans la quatrième et dernière étape, je traiterai de la question de l'autocritique de la raison éclairée kantienne, en tant qu'elle se développe finalement comme critique épistémologique s'inspirant de l'ancien scepticisme et intégrant son principe d'antinomie dans la conception métaphilosophique de la raison.

\section{La critique des Lumières}

Dans ses écrits de jeunesse, c'est-à-dire pendant les années 1785-1800, Hegel développe une attitude critique inspirée des Lumières. Au cours des périodes de Stuttgart (1785-1788), Tübingen (1788-1793), Berne (1793-1797) et Francfort (1797-1800) ${ }^{4}$, Hegel exerce une critique dirigée contre la culture et les institutions traditionnelles qui aboutit à

\footnotetext{
${ }^{4}$ La première édition des manuscrits de jeunesse de Hegel a été publiée en 1907 par H. Nohl, Hegels theologische Jugendschriften, Tübingen, Mohr, 1907 (désormais Nohl). L'édition critique des écrits et fragments des périodes de Stuttgart, Tübingen et Berne est maintenant disponible dans l'édition de l'Hegel-Archiv: G.W.F. Hegel, Gesammelte Werke, Bd 1. Frühe Schriften. Teil 1. Hrsg. von Fr. Nicolin und G. Schüler, Meiner, Hamburg, 1989 (désormais GW1); Bd 3. Frühe Exzerpte. Unter Mitarbeit von G. Schüler hrsg. v. F. Nicolin, Meiner, Hamburg, 1991 (désormais GW3).
} 
l'émancipation de la domination exercée par l'autorité politique et religieuse ${ }^{5}$. En effet, Herrschaft est un des mots revenant le plus souvent dans ses écrits. Sa critique est celle de la domination. Hegel développe pourtant, durant ses années de jeunesse, une pensée critique qui a comme objet la réalité sociale et politique de son temps. Cette attitude critique est liée au scepticisme religieux du XVII ${ }^{\mathrm{e}}$ et $\mathrm{XVIII}^{\mathrm{e}}$ siècles et à la critique biblique. Spinoza, Reimarus, Hume, Gibbon sont autant d'auteurs qui influenceront l'approche hégélienne de la religion et de l'Écriture ${ }^{6}$. Hegel critique constamment l'autorité religieuse, la théologie dogmatique et l'effet de l'exercice de leur domination. Il n'y a presque aucun élément de la dogmatique traditionnelle qui n'ait pas été soumis à la plus sévère des critiques : du péché originel à la providence, en passant par la double prédestination, jusqu'à la trinité et à l'idée de l'unicité et de la personnalité divine de Jésus ${ }^{7}$. Hegel ne considère jamais la Bible comme la source d'une vérité révélée, mais plutôt comme un document historique, qu'il analyse à l'aide d'instruments psychologiques et philosophiques soutenus par une méthode historique naturaliste. La religion est analysée en tant qu'institution sociale, sans qu'aucune cause supranaturelle ne soit introduite, et d'un point de vue pratique et politique. Dans les phénomènes religieux, Hegel identifie parfois des préjugés liés à l'exercice de certaines formes de domination sociale, parfois l'anticipation de vérités morales entièrement accessibles à la raison. Le titre Écrits théologiques qui fut apposé par l'éditeur (H. Nohl) de la première édition de ses manuscrits (1907), est pourtant très trompeur, car en fait il s'agit plutôt d'écrits politiques antithéologiques caractérisés par un certain scepticisme religieux.

Mais son attitude critique renvoie aussi à sa lecture des philosophes du XVIII ${ }^{\mathrm{e}}$ siècle, qu'ils proviennent de l'Enlightenment (Gibbon, Hume, Ferguson ${ }^{8}$ ), des Lumières françaises

\footnotetext{
${ }^{5}$ Pour une interprétation dans ce sens des écrits de jeunesse de Hegel voir I. Testa, Hegel critico e scettico. Illuminismo, repubblicanesimo e antinomia alle origini della dialettica, Padova, Il Poligrafo, 2002.

${ }^{6}$ L’influence de Reimarus en ce qui concerne la critique hégélienne du judaïsme et de la communauté chrétienne peut être retrouvée dans Nohl, p. 273 et p. 373-374. Voir sur ce point Ch. Jamme, Ein ungelehrtes Buch. Die philosophische Gemeinschaft zwischen Hölderlin, und Hegel in Frankfurt 1797-1800, Bonn, Bouvier, 1983 , p. 206, 241-242, 281. Selon Karl Rosenkranz, le premier biographe de Hegel (cf. K. Rosenkranz, Hegels Leben, Berlin, 1844, p. 48), Hegel aurait lu pendant la période de Berne le Tractatus theologico-politicus de Spinoza un livre qui était présent dans la bibliothèque de Tschugg ou Hegel travaillait comme précepteur. Sur l'influence de Hume (dont la Natural History se ressent déjà dans un des premiers écrits de Tübingen, cf. GW1, Text 13, p. 78), en particulier en ce qui concerne la conception de la « religion du peuple [Volksreligion] » - une notion qu'on retrouve déjà dans l'essai du 10 août 1787 Sur la religion des Grecs et des Romains (GW1, Text 4) - voir Testa, op. cit., p. 30 et p. 52-68. Il semble certain qu'Hegel pouvait trouver dans la bibliothèque de Tschugg l'History of the decline and Fall of the Roman Empire (London, 1766-1788) de E. Gibbon (cf. K. Rosenkranz, op. cit., p. 60 ; H. Strahm, «Aus Hegels Berner Zeit. Nach bisher unbekannte Dokumenten » dans Hegel in der Schweiz (1793-1796), hrsg. v. H. Schneider und N. Waszek, Bern/Frankfurt, P. Lang, 1996, p. 287-316, en particulier p. 314), l'historien anglais de formation sceptique dont les analyses sur la décadence de l'empire romain et sur l'origine du christianisme ont été influencées par Bayle, Montesquieu, les Lumières et surtout par Hume. Sur l'influence de Gibbon en ce qui concerne l'interprétation hégélienne des premières communautés chrétiennes, cf. Ph. Muller, «Hegel und Gibbon. Oder wie der Philosoph dank der Lektüre des Historikers zu sich selbst findet»dans Hegel in der Schweiz, p. 145-171. Sur l'influence de Gibbon en ce qui concerne la critique républicaine du christianisme et la liaison dangereuse entre christianisme et domination voir Testa, op. cit., p. 30 et p. 52-58.
}

\footnotetext{
${ }^{7}$ Pour une analyse systématique cf. M. Brecht e J. Sandberger, «Hegels Begegnung mit der Theologie im Tübinger Stift », Hegel-Studien, 5, 1969, p. 76-79.

${ }^{8}$ Pour une analyse de l'influence du Scottish Enlightenment sur le jeune Hegel voir N. Waszek, The Scottish Enlightment and Hegel's Account of Civil Society, Dohrdrecht/Boston/London, Kluwer, 1988. Pour l'influence de l'Essay on the History of Civil Society de A. Ferguson sur le sujet de la dialectique de la vertu dans les écrits de Berne voir Testa, op. cit., p. 176-177 et 185-186
} 
(Rousseau) ${ }^{9}$ ou de la Spätaufklärung allemande (entre autres Meiners, Kästner, Sulzer, Eberhard, Garve, Nicolaï, et surtout Mendelsshon et Lessing) ${ }^{10}$. De ce dernier courant et de la Populärphilosophie allemande proviennent en particulier l'idée de l'éclairement du bas et celle de l'éclairement du peuple. Le fait qu'Hegel critique très souvent le caractère abstrait de la conception kantienne de la raison pratique ne doit pas être confondu avec une attitude hostile aux Lumières. Bien qu'Hegel ait probablement eu connaissance, à Stuttgart déjà, de certaines œuvres de Kant - je pense notamment à Was ist Aufklärung ? $^{11}$, , il a toujours gardé une certaine distance avec le philosophe de Koenisberg et, surtout, ne l'a jamais identifié à l'Aufklärung. En fait, la critique de la raison pratique kantienne qu'on retrouve dans les écrits de jeunesse est souvent conduite à partir d'un point de vue inspiré d'une conception plus sensible de la raison qui provient, notamment, d'autres sources de l'Aufklärung allemande, de l'Enlightenment et des Lumières françaises.

La question la plus importante à laquelle les écrits de jeunesse d'Hegel tentent de répondre est donc la suivante : comment peut-on définir théoriquement et en pratique une Aufklärung des gemeinen Männes - un éclairement (une éducation) de l'homme commun - et donc élargir, étendre les idéaux de la raison et de la liberté au delà du cercle étroit des savants? Hegel avait déjà évoqué cette question dans le journal qu'il tenait à Stuttgart ${ }^{12}$, où la question de l'éclairement de l'homme commun rejoignait celle de l'éclairement des rapports publics ( «öffentliche Verhältnisse »). Au cours des années suivantes, il revient constamment sur ce problème en essayant d'identifier l'obstacle empêchant l'élargissement de l'Aufklärung et qu'il faut lever. En fait, Hegel se demande comment serait possible l'intervention de la philosophie dans la sphère publique ainsi que la restructuration des relations sociales qui la configurent.

Dans ces fragments, Hegel s'inspire d'un modèle normatif et idéalisé des rapports publics grecs, qu'il pense comme ayant réalisé une structure transparente de circulation du savoir du bas vers le haut et du haut vers le bas, circulation qui était possible parce que, dans le monde grec, la dimension sensible, ayant une forme libre et spontanée, rapprochait la multitude du peuple aux savants. Dans la perspective de ce modèle normatif des rapports publics grecs, Hegel en vient à diagnostiquer la pathologie principale de la société de son temps. La manifestation cognitive de la domination est liée à la manipulation de la sensibilité par la religion chrétienne. La réforme de la sensibilité, donc la réforme et la rationalisation de la religion, sont vues comme les moyens d'étendre publiquement la raison. On trouve ici un autre thème typique des Lumières, et notamment de l'Aufklärung allemande, qui a fait de la

\footnotetext{
${ }^{9}$ L'influence de Rousseau sur la notion de Volksreligion qu'Hegel avait déjà développée à Stuttgart pourrait être tirée du chapitre VIII du troisième livre du Contrat social où il est question de la « religion civile » (cf. M. Fujita, «Philosophie und Religion beim jungen Hegel », Hegel-Studien, Beiheft 26, Bouvier, Bonn, 1985, p. 33).

${ }^{10}$ Sur ce point sont très importantes, outre les annotations du Journal de Stuttgart, les Exzerpta de la période 1785-1788, qui témoignent de la lecture faite par Hegel de penseurs comme Kästner, Sulzer, Nicolai, Feder, Garve, Mendelssohn, Eberhard (et, à travers eux, de Voltaire, Gibbon, Montesquieu, Ferguson, Kant, Schiller, Rousseau). Cf. Frühe Exzerpte, in GW3, p. 1-200.

${ }^{11}$ On peut penser que la référence en partie ironique à «notre temps éclairé » retrouvée ou dans le Journal de Stuttgart (cfr. GW1, Text 1, p. 30) ou dans l'essai sur La religion des Grecs et des Romains, renvoie implicitement à la distinction tracée par Kant dans le Was ist Aufklärung? entre l' «époque des Lumières » (Zeitalter der Aufklärung) et les « époques éclairées » (aufgeklärte Zeitalter).

${ }^{12}$ GW1, Text 1, p. 30 .
} 
réhabilitation de la sensibilité l'un des ses traits les plus caractéristiques ${ }^{13}$ : on pense à Lessing, et à son Nathan, dans lequel le jeune Hegel voit le plus bel exemple d'une raison pratique à la fois éclairée et sensible ${ }^{14}$.

Le problème de l'élargissement de l'Aufklärung est à la base de l'interprétation hégélienne de la religion en général. Dans ses travaux de jeunesse, Hegel a en effet toujours suivi l'annotation déjà contenue dans le journal de Stuttgart selon laquelle : «l'éclairement de l'homme commun a toujours trouvé sa mesure dans la religion de son temps $»^{15}$. La religion est vue par Hegel d'une part comme l'expression de la domination, laquelle consiste en une manipulation de la sensibilité, et d'autre part, du fait qu'elle possède une très grande emprise sur la sensibilité, comme pouvant potentiellement devenir un moyen public de développement social de la raison, et donc d'éclairement.

En fait, à Tübingen Hegel se concentre sur la notion de religion populaire (Volksreligion) - une notion qui ne lui vient pas seulement de la «religion civile» de Rousseau, mais aussi de la notion de « religion of the vulgar » utilisée par Hume et reprise par Gibbon dans le sens de «true religion ${ }^{16}$. La religion populaire est entendue ici comme religion sensible compatible avec la raison et donc apte à réaliser une réforme de la sensibilité. Hegel n'a pas abandonné la critique de la religion positive, dont le point de vue n'est pas la religion mais plutôt l'idéal rationnel. En fait, l'esquisse d'une religion à la fois populaire et rationnelle est la continuation du projet de l'élargissement de l'Aufklärung et de la critique sceptique du dogmatisme religieux.

Dans les écrits de Berne, réunis par Nohl sous le titre La positivité de la religion chrétienne ${ }^{17}$, Hegel développe une critique du christianisme en tant que forme religieuse liée, dans la vie moderne, à la domination. Pourant, dans l'écrit sur La vie de Jésus ${ }^{18}$, il identifie néanmoins un contenu rationnel à la prédication de Jésus, lequel est vu comme un penseur des Lumières - un prophète de l'autonomie de la raison - qui à échoué dans son projet d'étendre l'Aufklärung.

Hegel a suivi la Révolution française dès le début tout en soutenant, au moins théoriquement, les événements qui y furent liés ${ }^{19}$. Avant 1789, Hegel avait déjà une attitude

\footnotetext{
${ }^{13}$ Sur ces aspects de l'Aufklärung allemande, voir P. Kondylis, Die Aufklärung im Rahmen des neuzeitlichen Rationalismus, Stuttgart, Klett-Cotta, 1981, p. 19.

${ }^{14}$ L'influence de Lessing, en particulier en ce qui concerne l'appréciation hégélienne du Nathan, se retrouve déjà dans la critique de l'imagination déformée développée par Hegel dans les premiers écrits de la période de Tübingen : cf. GW1, Text 15, p. 81 ; Text 16, p. 94.

15 «Sonst glaube ich auch, diese Aufklärung des gemeinen Mannes habe sich immer nach der Religion seiner Zeit gerichtet», GW1, Text 1, p. 30 .

16 Sur le rapport entre Gibbon et Hume, voir M. Andreas Weber, David Hume und Edward Gibbon, Religionsoziologie in der Aufklärung, Frankfurt am Main, Anton Hain Verlag, 1980. Sur la relation entre Hegel, Gibbon et Hume voir aussi Testa, op. cit., p. 101 sq.

${ }^{17}$ Cf. Nohl, p. 152-213 ; GW1, Texte 32-34, p. 279-378.

${ }^{18}$ GW1, Text 31, p. 207-278.

${ }^{19}$ Sur l'importance permanente de la Révolution française pour la pensée de Hegel, cf. Ritter, Hegel und die französiche Revolution, Frankfurt am Main, Suhrkamp Verlag, 1965; O. Pöggeler, "Philosophie und Revolution beim jungen Hegel » in O. Pöggeler, Hegels Idee einer Phänomenologie des Geistes, Freiburg/München, Alber,
} 
critique éclairée envers la religion et l'autorité; avec l'événement révolutionnaire, son orientation se précise historiquement - comme on peut bien le voir dans sa correspondance en répondant à l'exigence d'appliquer les idéaux de la raison et de la liberté à la réalité du temps, c'est-à-dire à l'Allemagne et à ses structures sociales, juridiques et politiques encore partiellement féodales.

Cela mènera Hegel, au cours de la période bernoise, à voir la modernité comme une époque spécifique, marquée par des structures déterminées - la forme juridique, la propriété privée, l'État moderne - lesquelles contiennent des éléments rationnels qui, s'ils ne se réalisent pas à un niveau supérieur, sont susceptibles de se retourner à leur tour en nouveaux moyens de domination. Hegel articule une conception spécifique de la domination moderne et de son lien avec la religion chrétienne : une conception qui doit expliquer l'origine et le déploiement de la scission du savoir social qui a conduit, en France, à son éclatement violent mais qui est, au même moment en Allemagne, encore intact et unifié. La critique devient celle du christianisme en tant qu'il est la religion qui définit l'époque succédant à l'antiquité grécoromaine. Selon cette forme de scepticisme religieux envers l'autorité dogmatique judéochrétienne, l'affirmation du christianisme, qui était au début l'expression d'une instance rationnelle d'autonomie, s'est développée plutôt sous la forme hétéronome et positive d'une auto-aliénation de la vie publique, un renoncement du peuple à l'exercice de l'autodétermination ${ }^{20}$. La réglementation de la vie intérieure selon les préceptes du christianisme devient le moyen de l'extension de la domination dans la vie privée de l'homme $^{21}$. On assiste donc à l'émergence d'une critique conduite à partir d'un point de vue inspiré par le scepticisme républicain - Gibbon et peut-être Machiavel influencent l'approche hégélienne ${ }^{22}$ - à l'égard du rôle public de la religion chrétienne.

\section{Les dialectiques des Lumières}

Même si le jeune Hegel est toujours resté fidèle à ses idéaux de raison et de liberté, il a néanmoins développé une vision complexe de l'Aufklärung, soutenue par une conception non linéaire du progrès. Entre Tübingen et Berne, Hegel commence à penser le chemin de la raison comme un «destin ${ }^{23}$, c'est-à-dire comme un processus qui, même si ses termes sont problématiques et parfois tragiques, achève l'élément irrationnel. L'expérience de la Révolution française et de ses évolutions intérieures, qu'Hegel voit à partir de son observatoire allemand, de même que ses études sur l'histoire du christianisme, le conduisent à penser que le chemin de la raison est constellé de dynamiques contradictoires. Le chemin de la raison sociale - et même de la raison qui s'affirme dans la société à travers la religion en tant que moyen et mesure de l'éclairement de l'homme commun - est parsemé d'effets de retournement par lesquels une instance rationnelle aboutit à son contraire, c'est-à-dire à la domination à laquelle elle voulait pourtant échapper. On trouve ici les premières structures

1973, p. 13-78; J. Habermas, «Hegels Kritik der Französischen Revolution», in Theorie und Praxis, Neuwied/München, 1967, p. 128-147.

${ }^{20}$ Cf. GW1, Text 26, p. 164.

${ }^{21}$ Cf. GW1, Text 32, p. 342-345.

${ }^{22}$ Voir Testa, p. 113 sq.

${ }^{23}$ Cette conception de la raison comme « destin » se trouve déjà dans GW1, Text 25, p. 159. 
dialectiques dans les reconstructions historiques d'Hegel. Il faut parler de ces dialectiques au pluriel, car Hegel découvre différents phénomènes de retournement des Lumières - ne disposant pas encore d'une théorie unificatrice ni d'une notion explicite de «dialectique »sans que cela doive signifier pour autant l'abandon du point de vue rationnel de la critique.

À Tübingen déjà, Hegel avait été frappé par le fait que l'affirmation de la raison, en tant qu'elle nie abstraitement son caractère pathétique et ses racines sensibles, s'accompagne souvent d'une nostalgie aiguë du mythe. On trouve ici le premier niveau de la dialectique des Lumières. Hegel réfléchit sur le rapport entre raison et sensibilité ainsi que sur le phénomène de la raison qui, en se comprenant comme entendement [Verstand] abstrait, finit par se retourner en sensibilité irrationnelle. Hegel conçoit alors comment le phénomène du rationalisme se mue en fétichisme de la raison, par exemple chez ceux qui collectionnent des reliques ayant appartenu à Rousseau ou à Frédéric le Grand ${ }^{24}$. En cela, la raison éclairée manifeste une persistante nostalgie d'un mythe qu'elle a elle-même dévoilé. Selon Hegel, si des traces de la sensibilité et de l'imagination doivent se manifester à chaque niveau des progrès de la raison sociale - la raison ayant une racine sensible - il s'agit donc de les réformer et de les discipliner plutôt que de les nier abstraitement. Voilà pourquoi il déclare qu'une religion populaire compatible avec les idéaux rationnels pourrait devenir un moyen d'éclairement de la sensibilité et de son émancipation de la domination.

On trouve la deuxième couche des dialectiques des Lumières dans les manuscrits hégéliens allant de la période de Tübingen à celle de Berne. Il ne s'agit plus ici du retournement de la raison en son contraire, mais plutôt d'un retournement intérieur, sur soimême, de la rationalité et du projet des Lumières. Hegel critique les «éclaircissements de l'entendement [die Aufklärung des Verstandes] », dont il écrit qu'ils nous « rendent certes plus ingénieux, mais non meilleurs $»^{25}$. Il faut dire qu'il ne critique pas l'entendement en tant que tel, mais plutôt certains usages qui réduisent l'entendement à un instrument, indifférent aux fins de la raison autonome. Si l'éclairement est conçu et pratiqué comme un simple moyen ou instrument, alors l'entendement peut devenir le moyen de n'importe quelle fin, et même de la logique de la domination dont il était censé nous émanciper. Cette critique de l'entendement conçu comme «froid, calculant, qui, oubliant son but, reste attaché à ses moyens $»^{26}$ est conduite pourtant à partir du point de vue d'un éclairement supérieur - eine höhere Aufklärung, selon les mots de Hölderlin, l'ami de Hegel qui, à cette époque, partageait beaucoup de ses conceptions - et à partir de l'exigence d'une émancipation de la domination. Hegel écrit à de nombreuses reprises dans ses manuscrits et dans sa correspondance avec Schelling que la théologie dogmatique de son temps essaie de se renouveler à travers l'entendement - par exemple à travers les moyens conceptuels du criticisme kantien, comme en témoigne le scepticisme fidéiste de leurs enseignants Flatt et Storr au collège théologique afin de donner à son édifice dogmatique l'apparence d'une rationalité ${ }^{27}$. Cette critique du scepticisme fidéiste en tant qu'usage apologétique du doute et de l'entendement se lie à la dialectique des Lumières, laquelle dévoile la tendance de l'entendement éclairé à devenir un

\footnotetext{
${ }^{24}$ Cf. GW1, Text 19, p. 124-125.

${ }^{25}$ Cf. GW1, Text 16, p. 94, tr. Fr. in G.W.F. Hegel, La vie de Jésus, précédé de Dissertations et fragments de l'époque de Stuttgart et de Tübingen, textes réunis et coordonnés par A. Simhon, Paris, Vrin, 2009, p. 64.

${ }^{26}$ GW1, Text 37, p. 407.

${ }^{27}$ Cfr. Hegel an Schelling, Ende Januar 1795, in Briefe von und an Hegel, I, hrsg. v. J. Hoffmeister, Hamburg, Meiner, 1969, Nr. 8, p. 16-17.
} 
simple instrument indifférent aux buts universellement humains, potentiellement réductible à un moyen de domination.

$\mathrm{Au}$ cours de la période de Berne, Hegel développe une première conception de l'histoire. On a vu qu'il comprenait différents phénomènes historiques - par exemple le christianisme et l'état moderne - comme des instances originairement rationnelles devenues, au cours de l'histoire, instruments de domination. Il faut voir ici que la question qu'Hegel se pose à propos de Jésus - comment a t-il été possible que la prédication de Jésus, laquelle exprimait la protestation de la raison éclairée et autonome contre le légalisme hétéronome judaïque, ait donné lieu, au cours de l'histoire, à la religion chrétienne et ses institutions positives, dogmatiques et violentes ? - est la même qu'il se pose après 1793 à propos de la Révolution française : comment a-t-il été possible que la raison qui s'est exprimée dans la Révolution et dans la déclaration des droits de l'homme ait donné lieu à la Terreur ?

Cette dernière question soulève en effet le problème le plus troublant pour Hegel, car elle le conduit à interroger l'histoire du passé, notamment religieux du christianisme et du judaïsme, afin d'y trouver une réponse lui permettant d'identifier les sources historiques et métaphysiques du drame de son temps. Dès lors, Hegel développe un modèle de développement historique conçu comme alternance intérieure de la raison et de la positivité, qu'il utilise comme schème interprétatif pour ses analyses historiques. La positivité, pensée comme retournement de la raison éclairée sur elle-même, désigne maintenant l'hétéronomie, cette dépendance à quelque chose d'extérieur, dogmatiquement acceptée. Par ailleurs, la positivité n'est pas simplement vue comme quelque chose qui, de l'extérieur, attaque la raison, mais plutôt comme quelque chose qui provient très souvent d'un durcissement de la raison elle-même, de sa réduction à l'entendement instrumental asservi à la domination. Cette logique de la positivité, la tendance de la raison à se positiviser, devient dès lors la clé d'une herméneutique de l'histoire qu'Hegel applique à différents phénomènes, comme les débuts du christianisme, les hérésies médiévales, l'État moderne, la Réforme, la Révolution française... Il s'agit de la première et embryonnaire philosophie de l'histoire hégélienne. On trouve ici, justement, l'un des premiers usages significatifs du mot «contradictoire [wiedersprechender] », utilisé à propos de la contradiction entre l'universalité rationnelle de la prédication de Jésus - toujours vu en tant que sage éclairé - et la positivité de ses effets ${ }^{28}$. Cette contradiction est le moteur d'une vraie dialectique historique propre aux Lumières (et il faut être attentif au fait qu'Hegel pense les Lumières sur un mode métahistorique, en retraçant les manifestations éclairées bien au-delà du XVIII ${ }^{\mathrm{e}}$ siècle) : une contradiction active dans le processus par lequel les instances rationnelles de l'Aufklärung deviennent positives en se métamorphosant en instrument de domination.

\section{L'esprit d'unité et la logique de la domination}

À Francfort, Hegel explique cette dialectique de la positivité, du retournement de la raison en domination, en terme de «destin [Schiksal] » ${ }^{29}$. La notion de « destin » est pourtant le moyen conceptuel par lequel Hegel comprend, à un niveau plus haut et plus général, les mécanismes historiques du retournement. Il s'agit ainsi d'une catégorie déjà dialectique

\footnotetext{
${ }^{28}$ Cf. GW1, Text 32, p. 297.

${ }^{29}$ Cf. Nohl, p. 374. L'édition critique des écrits de Francfort n'est pas encore terminée. On fera pourtant référence, en ce qui concerne plusieurs écrits de cette période, à l'édition de Nohl.
} 
n'impliquant pas une conception irrationnelle de l'histoire, le problème d'Hegel étant précisément de comprendre le mécanisme du destin afin de le dépasser. Par ailleurs, l'explicitation du mécanisme du destin mène Hegel à identifier une structure logique et gnoséologique liée à la dialectique de l'Auflklärung. Ici, le scepticisme religieux du jeune Hegel rejoindra le scepticisme épistémologique qui se penche sur le problème du critère et de la connaissance.

Dans ses écrits de Francfort, Hegel expose une dialectique métahistorique des Lumières. Cet usage métahistorique de l'Aufklärung n'est pas du tout un anachronisme : en fait, dans le débat allemand sur l'Aufklärung, par exemple dans les écrits de Mendelssohn ou ceux de Kant sur la question Qu'est-ce que les lumières ?, qu'Hegel connaissait déjà très bien à Stuttgart, on parlait déjà, sur un mode métahistorique, d'Aufklärung et même d'époques éclairées (Aufgeklärte Zeitalter). Or, la pensée des écrits de Francfort se présente encore une fois comme une critique de la domination : la «vérité » de la raison est quelque chose de libre, «quelque chose que nous ne dominons pas et par quoi nous ne sommes pas non plus dominés » ${ }^{30}$ et cela parce que « ce dont on est dépendant ne peut avoir la forme de la vérité ». La raison, par son orientation vers la vérité et la liberté, est donc critique de la domination qui les nie. «Comment auraient-ils pu exercer la raison et la liberté, ceux-là qui n'étaient que dominants ou dominés? » se demande Hegel. Le destin est notamment la condition à travers laquelle on fait l'expérience de cette fausse alternative entre dominer ou être dominé : «dans la nécessité », écrit Hegel, «l'homme est fait objet et opprimé ou bien doit faire de la nature son objet et l'opprimer ».

Selon Hegel la condition de nécessité de la domination propre à ce qu'il appelle le destin peut être comprise comme le produit de ce qu'il appelle l' « esprit de l'unité », «l'unité de la domination [Einheit der Beherrschung] » ${ }^{31}$. Quel est cet esprit de l'unité ? Il y a, proprement dit, une racine anthropologique archaïque de cet esprit de l'unité, en tant qu'elle manifeste une rupture de l'unité originaire de la nature - qu'Hegel retrouve symbolisée dans les mythes grecs et hébraïques du déluge - qui conduit l'esprit, c'est-à-dire la subjectivité naissante, à réagir par la peur et l'angoisse à l'altérité de la puissante nature, et donc à essayer de la réduire en un opposé devant être dominé et nié. L'esprit s'oppose au tout de la multiplicité qu'il trouve devant lui et dont il cherche à prendre possession. Ainsi, «afin que l'homme puisse faire face à la nature devenue ennemie, la nature devait être dominée ${ }^{32}$. Abraham et Noé sont dans la mythologie biblique les représentants d'une telle attitude. Pour faire face à une nature regardée comme hostile et ennemie, ils finissent par nier toutes les relations belles, en se réduisant eux-mêmes à la simple autoconservation de l'existence animale: «une hostilité universelle ne laisse place qu'à une dépendance physique, une existence animale ${ }^{33}$ écrit Hegel, c'est-à-dire, « le vide besoin de conserver l'existence physique $»^{34}$, « qui ne peut donc être assuré qu'aux frais des autres $»^{35}$.

\footnotetext{
${ }^{30}$ Cf. Nohl, p. 254, traduction française in G.W.F. Hegel, Premiers écrits (Francfort 1797- 1800), textes réunis, introduits, traduits et annotés par O. Depré, Paris, Vrin, 1997, p. 194-195.

${ }^{31}$ Nohl, p. 243-244 (Premiers écrits, p. 176-177).

${ }^{32}$ Nohl, p. 374.

${ }^{33}$ Nohl, p. 250 (Premiers écrits, p. 189).

${ }^{34}$ Nohl, p. 252 (Premiers écrits, p. 192).

${ }^{35}$ Nohl, p. 250 (Premiers écrits, p. 189).
} 
L'esprit de l'unité, qui surgit de la peur archaïque face à l'altérité de la nature et essaie d'y réagir en la réduisant en objet de sa domination, en objet sans vie, se retourne sur luimême, et se réduit en simple naturalité animale. Cet esprit s'assimile à l'objet mort qu'il voit devant soi et finit par être dominé par la nécessité naturelle dont il voulait s'émanciper et se rendre libre. En faisant abstraction de tous ses liens naturels, de ces belles relations grecques, l'esprit de l'unité devient esprit de sacrifice. Il se sacrifie lui-même et tout ce qu'il y a de beau dans les relations naturelles, et échange ainsi la liberté avec la conservation de soi, donc reste lié à la nécessité. La logique de la domination, de l'alternative entre dominer et être dominé, se reproduit donc à tous les niveaux de cet esprit et se manifeste en tant qu'un destin auquel il ne peut échapper. Cette logique autodestructrice active dans la civilisation se manifeste dans le christianisme - qui n'échappe pas à la logique de l'émancipation dont il voulait s'émanciper en retombant finalement dans la positivité - et même dans les résultats les plus hauts de la raison moderne, comme la subjectivité cartésienne qui oppose le sujet pensant au monde entendu comme matière inerte ${ }^{36}$. À cet égard, la raison pratique kantienne (et fichtéenne ensuite) est en partie pour Hegel la plus haute manifestation du lien entre la raison et la liberté. La raison pratique kantienne conçoit d'ailleurs l'autonomie d'une manière qui n'échappe pas à l'esprit de l'unité mais pourtant à la logique de la domination.

Dans un fragment écrit dans les années 1798-1800, on retrouve plusieurs des traits dessinés jusqu'ici ${ }^{37}$. On peut y voir tout particulièrement et explicitement le lien entre la thématique du destin, le concept dialectique de «contradiction » et les problèmes de la critique de la positivité et de l'exigence d'étendre les Lumières en établissant une alliance entre le cercle étroit des savants et la multitude, ainsi que de l'éclairement de l'opinion publique. L'analyse de la négativité naturelle et de la positivité historique est entendue ici comme « la réflexion sur son destin $»^{38}$, c'est-à-dire comme l'effort conceptuel d'en comprendre la logique et de la dépasser. Le mécanisme du destin est, à proprement parler, la logique de la positivité, qui est vue comme une logique de la contradiction. C'est pour cela qu'Hegel écrit que «le sentiment de contradiction entre la nature et la vie subsistante est le besoin qu'elle soit levée $»^{39}$.

L'arrière-plan de ce fragment - qui est strictement lié à une critique de l'obsolescence de la situation allemande - est une fois de plus la Révolution française - « une vie meilleure a inspiré notre époque ${ }^{40}$ - dont Hegel veut sauver le contenu émancipatoire, en refusant dans le même temps la méthode violente du dépassement du destin : une méthode qui selon Hegel reste dans la logique réactive de l'esprit de l'unité et de la domination. La contradiction toujours croissante expose - sur le mode aporétique, on pourrait dire négatif - une dynamique dialectique immanente au monde moderne qui a des racines objectives en même temps qu'elle est liée au tortueux processus d'éclairement de l'esprit et de la subjectivité humaine. Dans La

\footnotetext{
${ }^{36}$ Cette critique implicite du cartésianisme se trouve en particulier dans le fragment Die Liebe (novembre 1797), cf. Nohl, p. 378.

37 Der immer sich vergrössernde Widerspruch, in G.W.F. Hegel, Gesammelte Werke. Bd 5. Schriften und Entwürfe 1799-1808. Unter Mitarbeit von T. Ebert hrsg. von M. Baum und K.R. Meist, Meiner, Hamburg, 1998, p. 16-18 (Premiers écrits, p. 362-365).

${ }^{38}$ GW5, p. 16 (Premiers écrits, p. 363).

${ }^{39}$ GW5, p. 17 (Premiers écrits, p. 363).

${ }^{40}$ GW5, p. 17 (Premiers écrits, p. 364).
} 
contradiction toujours croissante, Hegel soumet la raison des Lumières et ses effets pratiques - notamment la révolution - à une autocritique afin de les libérer de leur forme encore asservie à la domination et à la violence. Cette critique est immanente en tant qu'elle ne soumet pas l'objet critiqué à des critères extérieurs, mais mesure plutôt l'adéquation entre cet objet et le critère qu'il se donne. Cette forme de critique immanente ou dialectique, en tant qu'exposition de la contradiction entre l'objet et son propre critère, se lie déjà en cela au scepticisme épistémologique ancien et à la question du critère. La critique sceptique des déterminations positives est déjà le modèle implicite de la critique hégélienne de la positivité historique et sociale; comme la positivité peut se développer dialectiquement de la raison même, il faudra que le scepticisme soit intégré dans la raison en tant que son moment négatif.

\section{Autocritique de la raison et scepticisme épistémologique ancien}

$\mathrm{Au}$ cours de la période de Francfort, Hegel développe une forme d'autocritique dialectique de l'Aufklärung ${ }^{41}$. Hegel voit maintenant dans le criticisme de Kant l'expression d'une forme d'Aufklärung qu'il faut soumettre à une critique immanente. Il faut être ici très attentif, car Hegel ne critique jamais l'Aufklärung en tant que tel, mais plutôt les formes déterminées de manifestation de la raison éclairée ; en l'occurrence, il n'identifie pas Kant à l'Aufklärung. La conception kantienne de la raison pratique doit être menée vers la réflexion sur elle-même et sur l'inadéquation entre son idéal d'autonomie - qu'il faut sauver et réaliser - et sa forme encore positive. C'est pour cela qu'Hegel dévoile la raison pratique kantienne, en tant qu'elle s'exprime sous la forme du devoir de la loi morale, comme toujours influencée par la logique de la domination étant donc destinée, au même titre que le christianisme, à se retourner en son contraire, c'est-à-dire dans l'hétéronomie d'une dépendance à la loi morale abstraite. Selon Hegel le devoir kantien renvoie à la même structure dualiste qui agit dans l'esprit de l'unité judaïque et ensuite chrétienne : une opposition entre unité et multiplicité, esprit et nature, loi morale et détermination sensible. L'homme vertueux de Kant s'émancipe de la constriction extérieure, de l'hétéronomie naturelle, en la transformant en force de coercition $^{42}$. Hegel voit ici une intériorisation de la logique de la domination plutôt que son élimination. Le principe de l'autodétermination rationnelle est certainement un moment d'éclairement et de progrès pratique, mais il est encore pris dans la forme de la domination et peut donc donner lieu à des retournements dialectiques.

Cette critique dialectique de la raison pratique de Kant, en montrant l'inadéquation entre son concept et sa réalisation, se poursuit dans l'esprit des Lumières et du scepticisme religieux, de l'affirmation de la raison et de la liberté contre le dogmatisme social et religieux.

\footnotetext{
${ }^{41}$ Pour une interprétation très différente de la dialectique de l'Aufklärung chez le jeune Hegel, par rapport à Rousseau et au sujet du mythe, voir notamment Ch. Jamme, «Aufklärung via Mythologie. Zum Zusammenhang von Naturbeherrschung und Naturfrömmigkeit um 1800 », in Idealismus und Aufklärung. Kontinuität und Kritik der Aufklärung in Philosophie und Poesie um 1800, Hrsg. v. Ch. Jamme und G. Kurz, Stuttgart, Klett-Cotta, 1988, p. 35-58 ; R. Bubner, « Rousseau, Hegel und die Dialektik der Aufklärung », in Aufklärung und Gegen Aufklärung in der europäischen Literatur, Philosophie und Politik von der Antike bis zur Gegenwart, Hrsg. v. J. Schmidt, Darmstadt, Wissenschaftliche Buchgesellschaft, 1989, p. 404-420.

${ }^{42}$ Dans ce sens Hegel parle de l' « autocontrainte [Selbstzwang] de la vertu kantienne » (Nohl, p. 290 ; Premiers écrits, p. 269) et montre que « la moralité est la dépendance à l'égard de moi-même, la division en soi-même » (Nohl, p. 390 ; Premiers écrits, p. 215).
} 
Il est vrai qu'Hegel critique parfois cette loi morale kantienne en lui opposant l'amour en tant que mode et expansion de la vie, mais il ne faut toutefois pas oublier qu'Hegel critique l'amour comme principe inadéquat d'intégration sociale à la communauté politique ${ }^{43}$, qu'il n'a jamais considéré l'amour en tant que tel comme une solution mais plutôt comme un analogon de la raison, c'est-à-dire comme l'expression, encore inadéquate, de la structure sensible de la raison reconnaissante ${ }^{44}$.

Finalement, la critique dialectique et réflexive de l'Aufklärung devient ici critique épistémologique. En effet, Hegel découvre à Francfort que la domination n'est pas seulement liée aux structures pratiques, mais aussi à des structures logiques et gnoséologiques ${ }^{45}$. La critique doit donc réfléchir sa forme et ses présupposés épistémologiques. La critique de la domination se mêle ici à la critique de la connaissance. Dans l'opposition entre unité et multiplicité, sujet et objet, esprit et nature, qui se manifeste à travers plusieurs phénomènes historiques et théorétiques, et enfin dans la raison pratique de Kant, Hegel découvre la structure gnoséologique du dualisme. Le scepticisme épistémologique joue ici un rôle très important. D'une part, Hegel lit Kant à partir du contexte de la crise sceptique du kantisme des années 1790. Déclenché par Garve et Schulze, ce débat révélant les conséquences sceptiques du criticisme - dans le sens du scepticisme moderne de Hume - est, selon Hegel, l'occasion qui permet le dévoilement des présuppositions dualistes de cette philosophie, tel qu'il l'écrira dans son article de 1802 sur Le rapport entre le scepticisme et la philosophie.

Hegel était familier de l'ancien scepticisme et du moderne - et ce dès les années de Stuttgart, à travers le scepticisme modéré de Meiners, Sulzer et Feder et l'approche pragmatique de l'histoire de Meiners et $S c h r o ̈ c k h{ }^{46}$ - de même qu'il se retrouva, pendant les années universitaires de Tübingen, dans un milieu intellectuel marqué par un renouveau de l'intérêt pour l'ancien scepticisme. Il faut rappeler que F.I. Niethammer, ami de Hegel et auteur, lors du séjour d'Hegel à Tübingen, d'une nouvelle traduction partielle des esquisses pyrrhoniennes $(1791)^{47}$ ainsi que C.F. Stäudlin, auteur de la Geschichte und Geist des Skeptizismus $(1794)^{48}$, étaient tous deux anciens élèves du collège théologique. Le plus célèbre enseignant du collège de Tübingen avait par ailleurs été G. Ploucquet, auteur d'une dissertation sur l'Epoché de Pyrron en $1758^{49}$. Au collège, Hegel a pu prendre conscience, à travers ses enseignants Storr et Flatt qui utilisaient la limitation kantienne de la raison en

\footnotetext{
${ }^{43}$ Dans ce sens, Hegel écrit que « l'élargissement contre nature de la sphère de l'amour s'embrouille dans une contradiction, dans un effort erroné qui devait devenir le père du fanatisme actif ou passif le plus terrible » (Nohl, p. 324 ; Premiers écrits, p. 314).

${ }^{44}$ Cf. GW1, Text 21, p. 133.

${ }^{45}$ Cette évolution épistémologique de la philosophie hégélienne se retrouve déjà dans les écrits de la fin 1797 et du début 1798, où l'opposition entre l'un et la multiplicité, l'universel et le particulier, la liberté et la nature, est pensée à nouveau à travers les catégories du « sujet» et de «l'«objet». Cfr. Nohl, p. 376-377 et 395.

${ }^{46}$ Voir Testa, op. cit., p. 46-50.

${ }^{47}$ F.I.Niethammer, Probe einer Übersetzung des Sextus Empirikus drei Büchern von den Grundlehren der Pyrrhoniker, in Beyträge zur Geschichte der Philosophie, hrsg. v. G.G. Fülleborn, Züllichau u. Freystadt, II, 1971, rist. anast. Bruxelles, Culture et Civilisation, p. 239.

48 C.F. Stäudlin, Geschichte und Geist des Skeptizismus, vorzüglich in Rücksicht auf Moral und Religion, Leipzig, 1794-1795.

${ }^{49}$ G. Ploucquet, Disputatio de Epoche Pyrrhonis, Tübingen, 1758.
} 
fonction d'un scepticisme fidéiste et d'un supranaturalisme ${ }^{50}$, du débat sur la crise sceptique du kantisme déjà ouvert par le compte rendu de la Critique de la raison pure publié par Garve en 1781 (où Kant avait été reconduit à Berkeley), renouvelé ensuite par AenesidemusSchulze, ce dernier ayant reconduit Kant à Hume (1792). À Berne, Hegel était devenu un lecteur assidu de ces penseurs aux tendances sceptiques comme Gibbon et Hume, et il a probablement lu la dissertation de E. Zeender De notione et generibus Scepticismi que Schelling lui avait demandé de lui envoyer par la poste ${ }^{51}$. De plus, selon le témoignage de Rosenkranz, Hegel pouvait acheter dans une librairie de Francfort les écrits de Sextus Empiricus et ceux de Platon ${ }^{52}$ : c'est d'ailleurs dès cette époque que l'on trouve dans les écrits de Hegel des traces de sa lecture du Parménide, le dialogue platonicien qu'il qualifiera, dans son article sur Le rapport entre scepticisme et philosophie (1802), comme le document le plus complet et autosuffisant d'un scepticisme authentique (dans lequel serait déjà contenu, selon Hegel, tous les tropes sceptiques antiques) ${ }^{53}$.

Le scepticisme religieux comme forme de la critique éclairée de la domination, et ensuite l'expérience des dialectiques des lumières, constituent donc le contexte dans lequel le scepticisme épistémologique devient pertinent pour $\mathrm{Hegel}^{54}$ en tant que réflexion sur le critère de la connaissance et sur la critique de la positivité et du dogmatisme, et en particulier ce dogmatisme qui est lié au scepticisme moderne à titre de dualisme et dont témoigne la philosophie kantienne. C'est ce qui mène Hegel à s'intéresser de plus en plus à la logique et à la théorie de la connaissance, sur lesquelles il n'avait pas encore vraiment écrit, et à s'intéresser au scepticisme antique comme au moyen le plus important pour critiquer les insuffisances des conceptions courantes et la positivité des déterminations dogmatiques liées à elles. L'effort de dépasser la crise sceptique de Kant et de l'Aufklärung avec les moyens pyrrhoniens est pourtant l'un des traits les plus marquants de la première philosophie hégélienne.

Dans les textes de Francfort, en particulier dans les fragments L'amour, foi et être (1797-98), et dans le Fragment de système de 1800, Hegel développe sa critique de la positivité religieuse et civile soutenue par les moyens épistémologiques des tropes sceptiques ainsi que par le principe de l'isosthénie/antinomie: principe utilisé, dans les écrits de Francfort, comme celui de la raison philosophique qu'Hegel, en 1802, qualifiera explicitement de vrai principe du scepticisme (et de la philosophie) authentique ${ }^{55}$. À Francfort, Hegel écrit, dans le fragment Foi et être, qu'il faut reconnaitre les déterminations dualistes et opposées comme «contraires, et leur rapport mutuel comme antinomie ${ }^{56}$. Au même titre il écrit, dans le Fragment de système de 1800, qu' " on peut montrer » à propos de chaque « expression », " en tant qu'elle est [quelque chose de] posé, que du fait que quelque

\footnotetext{
${ }^{50}$ À ce sujet voir K. Vieweg, Philosophie des Remis, op. cit., pp. 42-48.

${ }^{51}$ An Schelling, Januar 1796, Briefe, I, Nr. 16, p. 36.

${ }^{52}$ K. Rosenkranz, Hegels Leben, op. cit., p. 100.

${ }^{53}$ GW4, Verhältniss des Skeptizismus zur Philosophie, p. 207.

${ }^{54}$ Sur la distinction entre scepticisme religieux et scepticisme épistémologique, voir R.H. Popkin, The History of Scepticism from Erasmus to Spinoza, Berkeley, University of California Press, 1979.

${ }^{55}$ GW4, Verhältniss des Skeptizismus zur Philosophie, p. 208.

${ }^{56}$ Nohl, p. 382 (Premiers écrits, p. 137).
} 
chose est posé, en même temps [quelque chose d'] autre n'est pas posé, est exclu » ${ }^{57}$. La critique dialectique des Lumières se retourne ici en critique sceptique et épistémologique : en fait, la méthode d'analyse immanente des contradictions est dévoilée ici comme une nouvelle extension de la méthode sceptique de la comparaison de l'objet avec son critère, et de l'application de l'antinomie.

Le besoin de mener la raison kantienne, en tant qu'expression la plus avancée de la critique des Lumières, à réfléchir sur elle-même, peut, selon Hegel, être satisfait par le développement de la dialectique kantienne et de son principe antinomique - le cœur sceptique antique du criticisme - sur un mode débarrassé des présuppositions dualistes qui ont conduit Kant à retomber dans le scepticisme phénoméniste, ce qu'Hegel appellera quelques années plus tard (1801-02) le «fantasme du scepticisme [...] des temps modernes ${ }^{58}$. Dans ce sens Hegel écrira en 1801, dans ses Thèses d'habilitation, que le scepticisme est l'accomplissement du criticisme ${ }^{59}$, puis, en 1802, développe la thèse métaphilosophique selon laquelle le scepticisme antique, en tant que principe de l'antinomie, représente la face négative de la raison et de toute vraie philosophie ${ }^{60}$.

En effet, le scepticisme épistémologique n'exprime pas ici la destruction de la raison éclairée, mais plutôt son accomplissement, c'est-à-dire qu'il constitue le moyen négatif à partir duquel elle développe une réflexion sur elle-même et sur les critères d'un entendement critique, afin d'émanciper ce dernier de la forme dogmatique qui le pousse à retomber dans la domination. Si l'on se souvient de ce qu'Hegel écrira dans la Phénoménologie de l'esprit - à savoir que la critique des Lumières est l'exécution pratique de la négativité sceptique dans l'histoire $^{61}$ - et de ce qu'il écrira plus tard dans les Leçons sur l'histoire de la philosophie ${ }^{62}$ que les philosophies hellénistiques, dont l'ancien scepticisme serait le vrai accomplissement, sont l'authentique forme des Lumières grecques - alors la thèse selon laquelle la dialectique des Lumières trouverait en Hegel le lieu historique et théorétique où fut pleinement saisie l'importance du scepticisme dans sa signification religieuse, politique et épistémologique, n'est donc pas seulement une reconstruction postérieure à sa pensée, mais bien plutôt une clé de lecture qu'Hegel lui-même a voulu nous donner.

Italo Testa, Università degli studi di Parma

\footnotetext{
${ }^{57}$ Nohl, p. 347 (Premiers écrits, p. 372).

${ }^{58}$ GW5, Dass die Philosophie, p. 271.

59 «Philosophia critica caret Ideis, et imperfecta est Scepticismi forma » (GW5, p. 227).

${ }^{60}$ GW4, Verhältniss des Skeptizismus zur Philosophie, p. 208.

${ }^{61}$ Gesammelte Werke, Bd. 9: Phänomenologie des Geistes, hrsg. v. W. Bonsiepen und R. Heede, Hamburg, Meiner, 1980, p. 295.

${ }^{62}$ G.W.F. Hegel, Werke in zwanzig Bänden, hrsg. v. E. Moldenhauer u. K.M. Michel, Frankfurt am Main, Suhrkamp, 1971, Bd. XVIII, p. 359 ; Bd. XIX, p. 291, 320, 401.
} 